\title{
Park Assist: A Deep Convolution Neural Network based Parking Assistant system
}

\author{
Sukhdeep Sharma, Aayushya , Dr. Madhumita Kathuria, Pronika Chawla \\ Student, Department of Computer Science \&Engineering, \\ Manav Rachna International Institute of Research and Studies \\ Faridabad, Haryana, India \\ Assistant Professor, Department of Computer Science \&Engineering, \\ Manav Rachna International Institute of Research and Studies \\ Faridabad, Haryana, India \\ Assistant Professor, Department of Computer Science \&Engineering, \\ Manav Rachna International Institute of Research and Studies \\ Faridabad, Haryana, India
}

sharma.sukhdeep30@gmail.com,y.aayushya1998@gmil.com,madhumita.fet@mriu.edu.in pronika.fet@mriu.edu.in

Abstract - With the proliferation in number of vehicles an unnoticeable problem regarding parking of these vehicles has emerged in places like shopping complexes where current car parking facilities are incapable of managing the parking of vehicles without human labour . Even in current automated PGI's human labour is required in some or the other way. Motivated by the affordable and remarkable performance of Convolutional Nueral Network in various image classification tasks, this paper presents a review on the automated parking systems based on the CNN technique. The classifier are trained and tested by deep learning of nueral network thus using of PHP and HTML to

create the UI and knowledge of MySQL to create a database to store information about vehicles. Similarly by converting the process into three small procedures we will be able to evaluate the bill in accordance to the timestamp of the parked vehicle without the use of human efforts..

Keywords- Deep Learning, Convolution Neural Network, Image classification, Parking assistant

\section{INTRODUCTION}

With the evolution of technology parking system has made an drastic change. Various type of system have been in use. Every new phase of technology leads to more robust and effective system. This parking assistant is little part of the changing technology. In this system with the help Convolution Neural Network (CNN) which help to classify the multiple vehicles and provide the result with type of vehicle such as- Two wheeler, Four wheeler, six wheeler. This system is trained with large range of images with different vehicle type which help system to give more accurate result and hence make it more robust and accurate. The image classifying system helps to generate fully automated system[10].

Neural Network contains discrete units which are called neurons. They can optimised automatically by training. More they are trained more accurate results they give. They are stored as array of multiple neurons. Individual neuron is connected to its corresponding layer[2]. The data is transmitted from input layer to output layer through neurons. Individual vertex processes basic mathematic operations. And passes the result to its corresponding layer. The ending layer contains the loss function which linked with the classes. Pattern are recognized in CNNs is majorly in the pictures. This helps to embed the characteristics of the image into the structure, which initiate to Convolution Neural Network.

\section{I.I Convolutional Neural Network with Image Classification}

Convolutional Neural Network(CNN) was introduced by Yann LeCun in 1998. It includes some characteristics of neural network. This architecture is used in large amount in image classification. It is also used in major organisations such as Amazon which uses CNN for the section(suggestions for the product), Facebook uses it for tagging algorithm.

Here's a detailed operations of CNN in image classification. The key job of classification of image is acquiring an input and characteristics of image's class.

The perspective of humans that are developed throughout their life is way different than the way computer see an image. For ex.- the image of elephant seen by human and computer is different as shown:- 


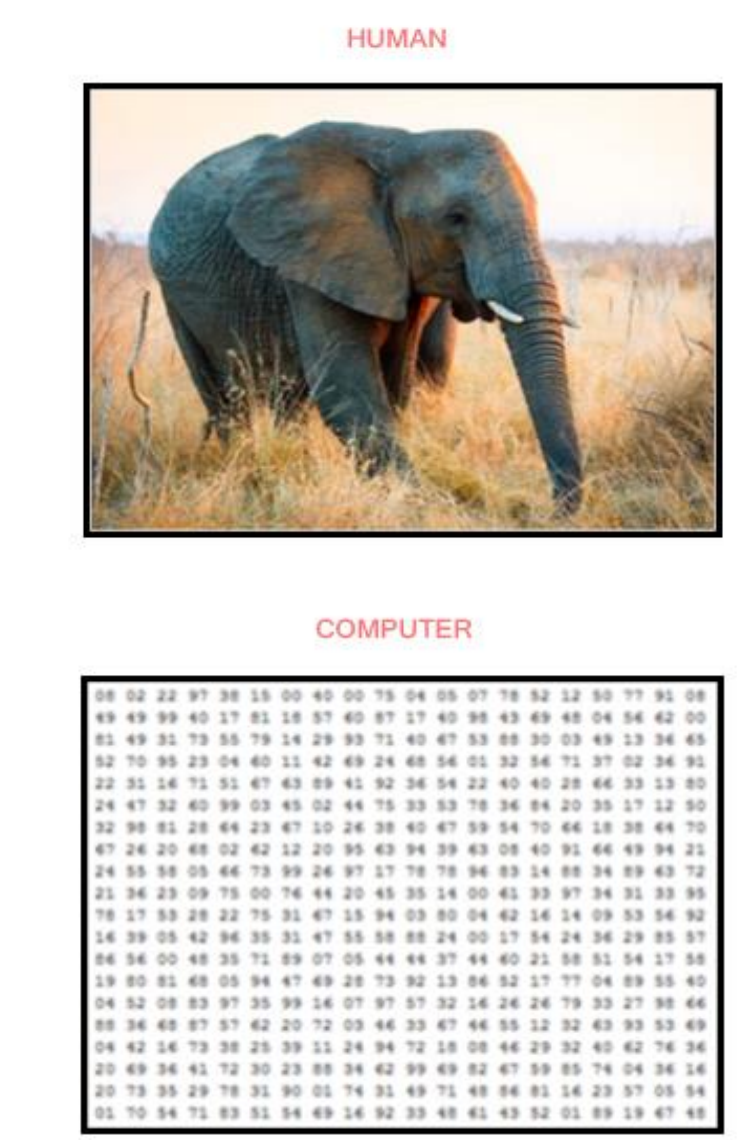

Fig 1 Computer interpretation of a picture

In the view of computer it's an array of pixels[3] than image. In the above image size is $300 \mathrm{X} 300$. Hence, size of an array will be $300 \times 300 \times 3$. Where it is arranged in order Width X Height X RGB channel values. The channel's value is between 0 to 255 is allocated to each of these numbers. The value defines the pixel's intensity at individual point. The base level definition of image is computed by computer to resolve the problem. As ears and legs are seen by humans are curvatures and boundaries for computer. And by all the layers details are analysed in more detail.[10]

\section{METHODOLOGY}

The whole working of the project takes places in three different procedures that contains the movement, identification and verification of different type of vehicle.

1. The Entry Point

2. The Image Processing

3. The Exit Point

ParkAssist: A Deep Convolution Neural Network based Parking Assistant system

\section{II.I The Entry Point Of Parking Procedure}

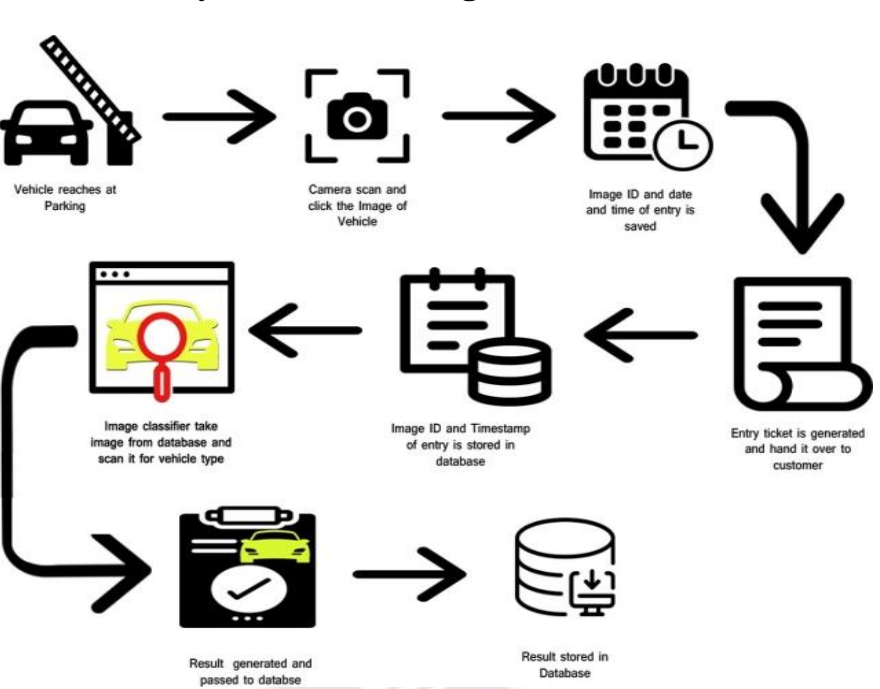

Fig 2 Flow chart of functionality at entry point of the procedure.

\section{Steps followed:}

The Entry Point is the starting phase of the three procedures. In this phase the vehicle enters the parking zone and its movement is topped by the barrier as soon as vehicle move enters the zone. The sensors senses the distance of the parking assistance system in accordance to the vehicle.

Vehicle enters the parking zone and is stopped by the barrier

The camera placed at the ceiling examines the vehicle by scanning it, clicking a picture and records its movement while entering. The image captured by the camera is passed through the image classifying technology designed and fitted in the assistance system.

\section{Camera captures the image}

As soon as the image is captured, an image ID is provided the picture clicked and the time of entry of vehicle is noted and passed on to the machine

\section{Image ID is generated and Time of entry of the vehicle is} noted

The time noted before is stored in the memory and a ticket is printed containing a bar code in which the vehicle information and time of entering the vehicle is encoded.

Entry ticket is generated and handed to the customer At the same time of generation of the ticket parallely the image ID and time stamp is entered into the database already generated before.

\section{Image ID and time stamped in the database}

The image classifier that uses the technology of Neural networks scans the image as it is stored in the database examines it by converting it into small pixels of the range appropriate enough to detect the image type or the type of vehicle whether it is two wheeler or four wheeler by 
comparing it to the set of features already specified in the input data stored in the database.

Image classifier take the image from the database and verify the vehicle type

The time and the image ID helps us to identify the type of vehicle as soon as the information of the specifications of the image is matched with the information already present in the database helping to identify the vehicle type.

Result generated and passed to the database

The result that has been generated is stored in the database until the vehicle appears again at the exit point.

\section{Result stored in the database}

The image ID and timestamp is stored in database.

\section{II.II The Image Classification}

Here, Image processed through sequence of multiple layers such as pooling, convolutional ${ }^{5}$, fully connected, non linear and then gives result.

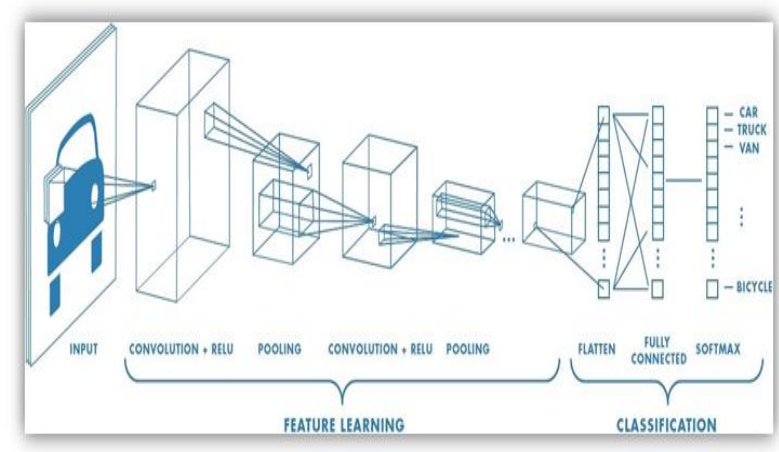

Fig 3 layers of image classification.

The initial layer is Convolutional layer.

The Image which is matrix with pixel values is inputed in the convolutional layer. The values are scaned at the top of left of image.

Then minor matrix is selected by the software. The minor matrix is called filter or neuron.

Then convolution is produced by filter, i.e. filter proceed with the image inputed, It's task is solve product of its value and image original pixel values. And all the calculations are done and single number value is obtained at last.[5]

Filter moves forward by a single unit from upper left corner and perform same operations at every preceding unit. When filtered is passed through all units, The resultant matrix is compacter than the matrix inputted.

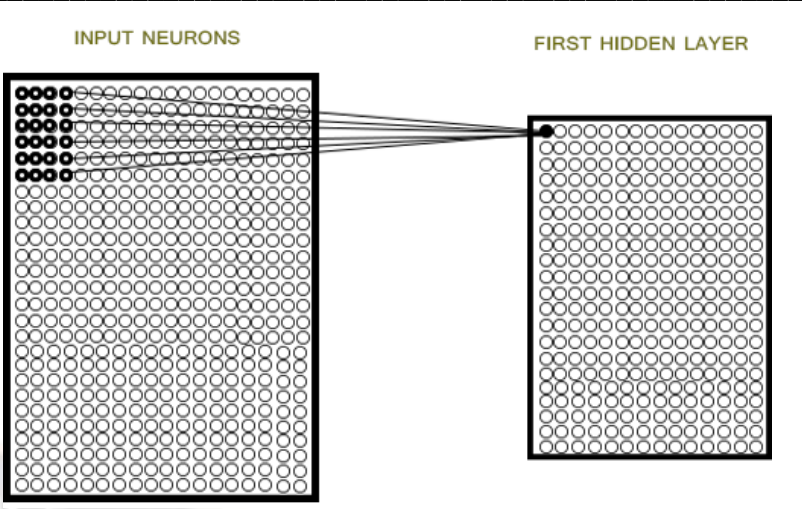

fig 4 Neurons into various layers.

As per the view of person it's just like recoganising edges and basic colors on the image. But if legs or face of an elephant (Higher level properties) is required to recognized the this network is required.

The network is combination of multiple convolution network and some layers like nonlinear and pooling layer. When input(image) is inputted in first layer i.e- convolution layer, then

preceding layer takes the output of first layer as it's input. This is observed in all the convolution layer in the network.

\section{The nonlinear layer}

This layer is added after every individual convolution calculations. It has an activation function, which brings nonlinear property. This property allow network to become considerably powerful and model the response variable.

\section{The pooling layer}

This layer comes after linear layer. It calculates dimentions(width and height) and processes down sampling calculations on them. Which leads to decrease in volume of an image. This explains that more deapth images are not used in processing as main features of it extracted in prior layers and intensity of image is decreased.[6]

When certain layers are processed like non-linear layer, pooling ad convolutional layers, nonlinear and pooling layers, then completely connected layer is required to coupled. The output obtained from the network ${ }^{5}$ is becomes the input of this layer. Dimensional vector(N) is obtained by coupling the fully connected layer to rear of the network. Here $\mathrm{N}$ is number of classes by which is model chooses the required clsses.

Some of the important phases for image classification are:-

1. Construction of model

2. Training a model

3. Testing a model

4. Evaluation of model 


\section{Construction}

At first model is constructed in which whole model functions and classes are defined. It is based on algorithms of machine learning. In this project, model is CNN.

\section{Training}

Model is explained above and then is trained using data, several images are used for training the model in which model become more robust. Then output according to image is trained. Represented as: model.fit(training_data,expected _data).[7]

\section{Testing}

After the training phase model is tested. In this phase another set of images is added. Hence it is new to the model so it helps to get the verification of accuracy.

\section{Evaluation}

Finally, Model is evaluated i.e.- Model is versatile to new data set.

\section{II.III The Exit Point of the Parking procedure.}

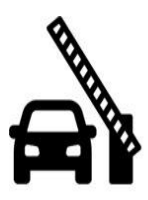

Vehicle reach exit point

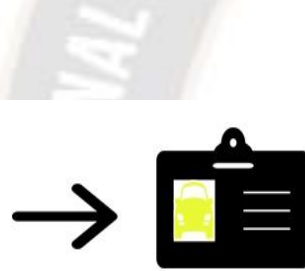

Image ID Verified

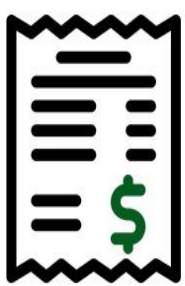

Parking Exit ticked is generated

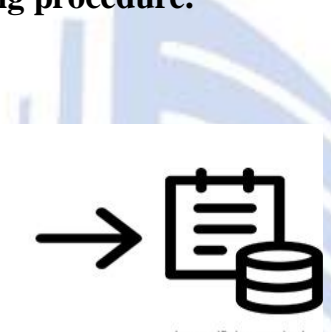

Image ID is searched in Database + Exit
Timestamp
Fig 5 Flowchart of functionality at exit point

\section{Steps followed}

The last procedure starts as soon as the parking slot is empty and the vehicle moves out towards the parking assistant system and the barrier stops it from going outside.

\section{The vehicle exits the parking slot}

The vehicle reached the parking assistant system machine and the barrier comes down. The customer submits the entry ticket to the assistance machine so that it reads the encrypted code on the ticket to give the exact amount to be paid.

\section{Image ID verified}

As soon as the ticket is submitted the stored information containing the type of database and image id is examined such that the entry time is mentioned helps to detect the amount to be paid. According to the exit time the total fare is to be calculated

ID is searched in the database and the exit timestamp The parking assistance generates an exit ticket with the entry time, exit time and the type of vehicle in correspondence to the total amount to be paid calculated according to the number of hours. The vehicle is not allowed to exit before the amount is paid. As soon as the bill is paid the barrier goes up allowing the vehicle to move out of the parking slot.

\section{Bill is paid and the vehicle moves out.}

\section{CONCLUSION}

To conclude we have created a system using the existing technology of Convolutional Neural network. In this project our main aim is to design a smart parking assistant that would detect the type of vehicle that is two-wheeler or fourwheeler using image classification at the time the vehicle enters the parking zone, after verifying the type of vehicle the ticket generator would generate the parking ticket mentioning the entry time and type of vehicle. Later at the time of exit, the ticket is examined with the time of entry and type of vehicle and the parking bill is generated accordingly . Thus providing an automated system for parking assistance using image classification techniques. Aim is to build strong foundation of team, technology, and partnerships with leading system integrators and parking equipment manufacturers enables it to provide a single seamless platform for all stakeholders involved like operators, consumers, equipment manufacturers, and city administrations.

\section{SCOPE AND FUTURE ENHANCEMENT}

The database can be expanded and more features can be added.

The automatisation of the system can be extended.

The cameras can be placed at the parking slots to specify perfect position of parking for vehicles entering the parking zone

Enhanced version of neural networks can to applied for object classification too.[8]

Result scan be made more accurate.

Aims to create future-proof parking technology and connect parking industry internally as well as with urban mobility players.

\section{ACKNOWLEDGEMENT}

The successful realization of the paper is an outgrowth of a consolidated effort of people from desperate fronts. I am thankful to Dr. Madhumita Kathuria (Assistant Professor) and Mrs. Pronika Chawla for her variable advice and 
International Journal on Recent and Innovation Trends in Computing and Communication

ISSN: 2321-8169 Volume: 8 Issue: 6

DOI: https://doi.org/10.17762/ijritcc.v8i6.5410

Article Received: 28 May 2020 Revised: 15 June 2020 Accepted: 22 June 2020 Publication: 30 June 2020

support extended to me without which I could not be able to complete my paper for a success.

\section{REFERENCES}

[1] O'Shea, Keiron \& Nash, Ryan.(2015). “An Introduction to Convolutional Neural Networks". ArXiv e-prints

[2] Datamind.cs [Online] .Available: "http://www. datam in d.cz/cz/Sluzby-Data-Science/u mela-intelingence-AI-MLmachine-learning-neural-net".

[3] Faheem, S.A. Mahmud, G.M. Khan, M. Rahman, H. Zafar " A Survey on car parking system" Journal of Research and Technology vol 11. Issue 5

[4] Parking net .com [online] Available "https://www.parkingnet.com/parking-industry-blog/get-my-parking/history-ofthe-automated-parking-system.

[5] Neha Sharma, Vibhor Jain , Anju Mishra "An Analysis of convolutional nueral network for image classification" ICCIDS 2018
[6] Deepaka Jaiswal , Sowmya V , KP Soman “ Image Classification of convolutional nueral network".

[7] Farhana Sultana , Abu Sufian, Paramartha Dutta "Advancements in Image Classification using Convolutional Neural Network" 2018 Fourth International Conference on Research in Computational Intelligence and Communication Networks.

[8] Mingyuan Xin1 and Yong Wang2 “ Research on image classification modelbased on deep convolution neural network".

[9] Muthukrishnan Ramprasath , M.Vijay Anand , Shanmugasundaram Hariharan " Image Classification using Convolutional Neural Networks".

[10] Debaditya Acharya , Weilin Yan , Kourosh Khoshelham “ Real Time Image Based Parking Occupancy Detection Using Deep Learning". 